\title{
Remote Episodic Memory Deficits in Patients with Unilateral Temporal Lobe Epilepsy and Excisions
}

\author{
Indre V. Viskontas, ${ }^{1}$ Mary Pat McAndrews, ${ }^{1,2}$ Morris Moscovitch ${ }^{1}$ \\ ${ }^{1}$ Department of Psychology, University of Toronto, and ${ }^{2}$ Neuroscience Program, University Health Network, Toronto \\ Western Hospital, Toronto, Ontario M5T 2S8, Canada
}

\begin{abstract}
The nature of remote memory impairment in patients with medial temporal lobe damage is the subject of some debate. While some investigators have found that retrograde amnesia in such patients is temporally graded, with relative sparing of remote memories (Squire and Alvarez, 1995), others contend that impairment is of very long duration and that remote memories are not necessarily spared (Sanders and Warrington, 1971; Nadel and Moscovitch, 1997). In this study, remote memory was assessed in 25 patients with unilateral temporal lobe epilepsy and 22 non-neurologically impaired controls using the Autobiographical Memory Interview (Kopelman et al., 1989). Results indicate that patients have impaired personal episodic memory but intact personal semantic memory. The impairment extends even to the most remote time periods in early childhood, long before seizure onset in many patients. As well, patients awaiting temporal lo-
\end{abstract}

The study of remote memory is key to understanding the role of the hippocampal complex in memory consolidation. The development of a standardized test, the Autobiographical Memory Interview (AMI), by Kopelman et al. $(1989,1999)$ has made it easier to assess personal remote memory reliably in humans. The AMI measures personal semantic and episodic memories from different time periods - childhood, early adulthood, and the recent past-permitting examination of the possible differential effects of brain damage on these two types of autobiographical memory.

In keeping with earlier observations (Ribot, 1882; Burnham, 1903; Scoville and Milner, 1957) Squire (1992) and Squire and Alvarez (1995) noted a temporal gradient to retrograde amnesia (RA) associated with medial temporal damage with the most remote memories differentially spared. He concluded that the hippocampus is involved in the establishment of new memories and their temporary storage but that over time these memories become independent of the hippocampus through consolidation. Once consolidation is complete, the memory trace resides elsewhere, rendering the hippocampus unnecessary for retrieval. According to consolidation theory, patients with temporal lobe epilepsy (TLE) and damage to the hippocampal system, should show a temporal gradient on remote memory tests. Furthermore, damage to this region should have a similar effect on all forms of declarative memory with no distinction among episodic and semantic memories, assuming no differential rehearsal.

Nadel and Moscovitch (1997) describe an alternative theory of

Received Dec. 22, 1999; revised May 5, 2000; accepted May 9, 2000.

This work was supported by Grant A8347 from the Natural Sciences and Engineering Research Council of Canada (M.M.) and by the Clinical Neuroscience Research Fund of the University Health Network (M.P.M.). For their contribution and time, we thank the patients and control participants. We also thank Stephen Taylor for help with the statistical analysis and Mary Jaciw for assistance in compiling psychometric data and scoring for analysis of inter-rater reliability. This research was completed in partial fulfillment of requirements for an undergraduate thesis at the University of Toronto.

Correspondence should be addressed to Mary Pat McAndrews, Neuroscience Program, EC2-009, Toronto Western Hospital, 399 Bathurst Street, Toronto, Ontario M5T 2S8, Canada. E-mail: mcandrws@uhnres.utoronto.ca.

Copyright (C) 2000 Society for Neuroscience $0270-6474 / 00 / 205853-05 \$ 15.00 / 0$ bectomy for control of seizures perform as poorly as those who have already undergone resective surgery. These results support the hypothesis that temporal lobe damage or dysfunction, caused by recurrent seizures or surgical excision, results in extensive retrograde amnesia for personal episodic memories. Interestingly, patients with radiological evidence of hippocampal sclerosis were not significantly more impaired than those without obvious sclerosis. These results indicate that even minimal damage to medial temporal lobes results in significant impairment to autobiographical episodic memory. These findings are more compatible with a memory loss or retrieval deficit rather than a consolidation account of remote memory impairment.

Key words: remote memory; episodic memory; epilepsy; temporal gradient; multiple trace theory; consolidation; autobiographical memory hippocampal function called the multiple trace theory (MTT). According to MTT, the hippocampal complex rapidly binds novel information and experience into a coherent memory trace composed of the hippocampal elements active at the time of encoding and the neocortical (or other) neurons that represent the events' features or components. Each episodic (autobiographical) memory trace consists of this ensemble of neurons as long as it exists. No long-term consolidation process is postulated in which the hippocampal contribution is relinquished. The reactivation of a memory trace establishes a new representation that shares some or all of the information in the original trace. The creation of multiple episodic traces lays down an associative network of related information and initiates the formation of semantic memory stores. These stores are based on extracts of factual information from the episodes and can be coded independently from them. Because MTT postulates that the hippocampal complex is an integral part of the autobiographical memory as long as it exists, damage should lead to loss of even remote episodic memories.

According to this account, the probability of recalling a remote event depends on the number of traces that code relevant information. Because it is unlikely that autobiographical episodes will be recovered in full detail, fewer complete traces will be laid down for them than for personal semantic information. As a result, after hippocampal complex lesions, the temporal gradient is expected to be shallower and extend back further in time for autobiographical episodes than for semantic memories. Our study set out to test the contrasting predictions made by these theories regarding loss of episodic and semantic memory after medial temporal lobe damage.

\section{MATERIALS AND METHODS}

\section{Participants}

Twenty-five patients with TLE participated in the study. Eleven patients had seizures originating in the right temporal lobe, five of whom were being assessed for resective surgery, and six of whom had already undergone surgery. Fourteen patients had seizures originating in the left temporal lobe, eight of whom were being assessed for surgery, and six of whom had already undergone surgery. All patients had documented damage involving the medial temporal region. In the preoperative group, there was 1 case with tumor, 2 with cavernomas, and 10 with radiological evidence of 
Table 1. Mean age and years of education for patients and controls

\begin{tabular}{llllll} 
& \multicolumn{2}{l}{ TLE patients } & & Controls \\
\cline { 2 - 2 } & Mean & $(\mathrm{SD})$ & & Mean & $(\mathrm{SD})$ \\
\hline$n$ & 25 & & 22 & \\
Age (years) & 37.7 & $(9.95)$ & & 35.0 & $(9.27)$ \\
Education (years) & 13.7 & $(2.20)$ & 13.8 & $(3.05)$ \\
\hline
\end{tabular}

Table 2. Seizure variables and psychometric data for patients

\begin{tabular}{|c|c|c|c|c|}
\hline & \multicolumn{2}{|c|}{ L-TLE } & \multicolumn{2}{|c|}{ R-TLE } \\
\hline & Mean & $(\mathrm{SD})$ & Mean & $(\mathrm{SD})$ \\
\hline MTS (percent of sample) & 55 & & 57 & \\
\hline Seizure onset (age in years) & 10.9 & $(7.73)$ & 15.8 & $(12.40)$ \\
\hline WAIS-R/III full-scale IQ & 89.3 & $(8.37)$ & 94.9 & $(15.38)$ \\
\hline Delayed recall stories $(z)$ & -0.72 & $(0.54)$ & -0.47 & (1.11) \\
\hline Delayed recall figures $(z)$ & 0.42 & $(0.79)$ & -0.56 & $(1.26)$ \\
\hline
\end{tabular}

MTS, Medial temporal sclerosis as assessed by radiologist.

mesial temporal sclerosis. Cases with surgical resections involved excision of the amygdala and $1-2 \mathrm{~cm}$ of hippocampus. Three patients had pathology in additional areas independent of their seizure focus; one with a small region of encephalomalacia in the left occipital pole, one with atrophy of the right thalamus, and one with cavernomas in the left thalamus/insula. All patients that had undergone surgery were either seizure-free postoperatively or had a $75 \%$ reduction in seizure frequency. Those tested postoperatively were seen at least 5 months and at most 8 months after surgery.

Twenty-two non-neurologically impaired controls matched for age and education also participated. All participants gave informed consent, and controls were paid $\$ 20.00$ each for their participation. Control participants had no history of epilepsy or other neurological disease and were recruited using advertisements in the hospital, whereas patients were asked if they would like to participate by the neuropsychologist conducting their clinical assessment. Patients and controls were all right-handed, with the exception of one control (LS). There were 12 female and 13 male patients and 11 female and 11 male controls.

Demographic information for participants is presented in Table 1; patient and control groups were matched for age and years of education. Table 2 displays relevant psychometric data for patients, including fullscale intelligence quotient (Weschler Adult Intelligence Scale-Revised or Weschler Adult Intelligence Scale-III) and standardized scores ( $z$ scores $)$ representing delayed recall from WMS measures of paragraph recall and visual reproduction. The latter measures were selected to characterize anterograde memory functioning in our patient sample. Because different versions of the Weschler Memory Scale (WMS-R and WMS-III) were used across patients, $z$ scores were calculated based on normative reference data provided in test manuals. The proportion of patients who had mesial temporal sclerosis by radiological criteria and the mean age of seizure onset are also displayed in Table 2.

\section{Materials and design}

Administration and scoring procedures as described in the AMI tes manual (Kopelman et al., 1989) were followed. The interview consists of two types of questions, administered concurrently: (1) personal semantic questions, and (2) autobiographical incidents questions. The questions survey three distinct time periods: childhood (ages $0-18$ ), early adulthood (ages 18-30), and recent (within the past 5 years). As well, the early time period can be further subdivided into three time periods: ages $0-5$, ages 5-11, and ages 11-18.

Personal semantic questions. Subjects were asked questions relating to their personal past, including names and locations of schools attended, home addresses, and names of friends. Each time period had a maximum score of 21 points.

Autobiographical incidents questions. Subjects were asked to relate incidents that occurred during each of the three time periods and to give temporal and spatial contextual information for each incident described Three such incidents were probed for each time period, and specifications such as "first day at work" were used as probes. Responses were recorded on the scoring sheets as close to verbatim as possible. Each incident was scored out of a possible score of 3 , based on the descriptive richness and specificity in time and place of the response. The maximum score per time period was 9 . One rater scored each of the questions, and an independent rater who was blind to subject group and original ratings scored $25 \%$ of the episodic items.

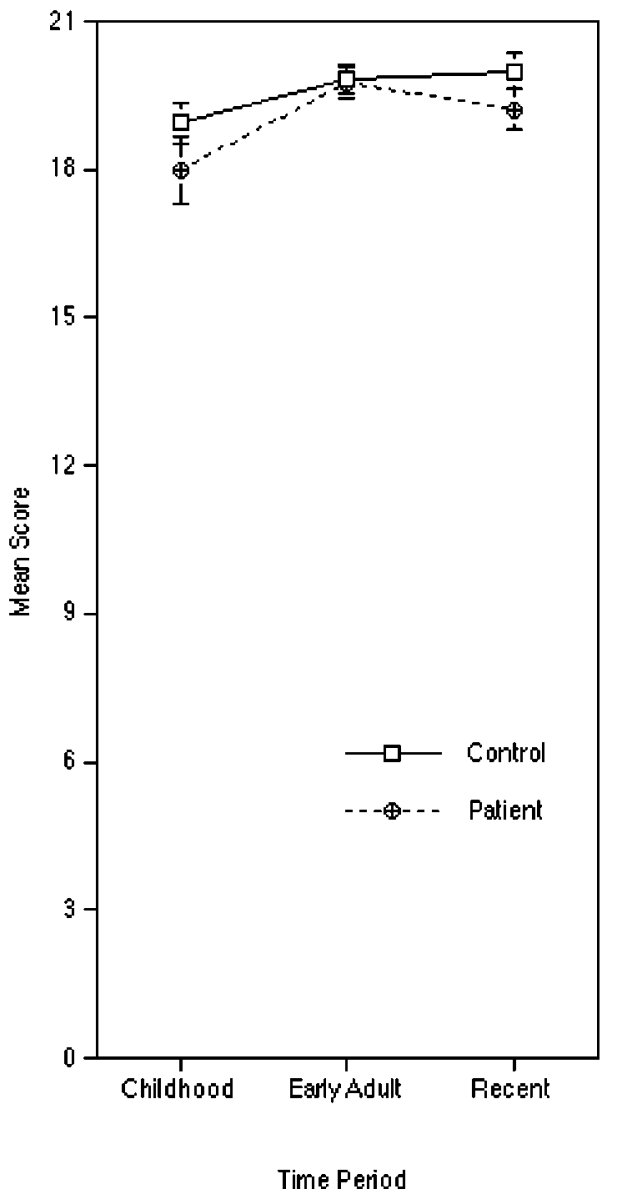

Figure 1. Personal semantic memory performance. Mean scores on semantic components of AMI for control $(n=22)$ and patient $(n=25)$ groups. The maximum score is 21 per time period. Vertical lines depict SEM values.

\section{RESULTS}

\section{Participants}

There were no significant differences between groups for age or education $(p>0.05)$. TLE patients demonstrated mild memory deficits on the WMS, with left TLE patients showing selective verbal recall impairment, whereas right TLE patients had equivalent difficulty with both verbal and nonverbal recall. A 2 (hemisphere $) \times 2$ (preoperative vs postoperative status $) \times 2$ (material) ANOVA revealed a significant side by material interaction $\left(F_{(1,20)}\right.$ $=7.575 ; p<0.02)$. Of note, there was no main effect or interaction involving operative status, indicating that those patients awaiting surgery showed equivalent anterograde memory impairments to those who had undergone temporal excisions.

\section{AMI}

Figures 1 and 2 display the mean scores for personal semantic and episodic questions by time period for patients and controls. Note that the two test components are on different scales because the total possible score for semantic questions is 21 and for episodic items is 9 for each time period.

\section{Inter-rater reliability}

An interclass correlation (Howell, 1997) was used to evaluate inter-rater reliability for responses scored by two individuals. The resulting coefficient was 0.75 , which compares favorably with those reported in the test manual (0.83-0.86).

\section{Overall ANOVA}

Proportions were used to compare episodic and semantic data, because these are based on different numbers of total points. An 


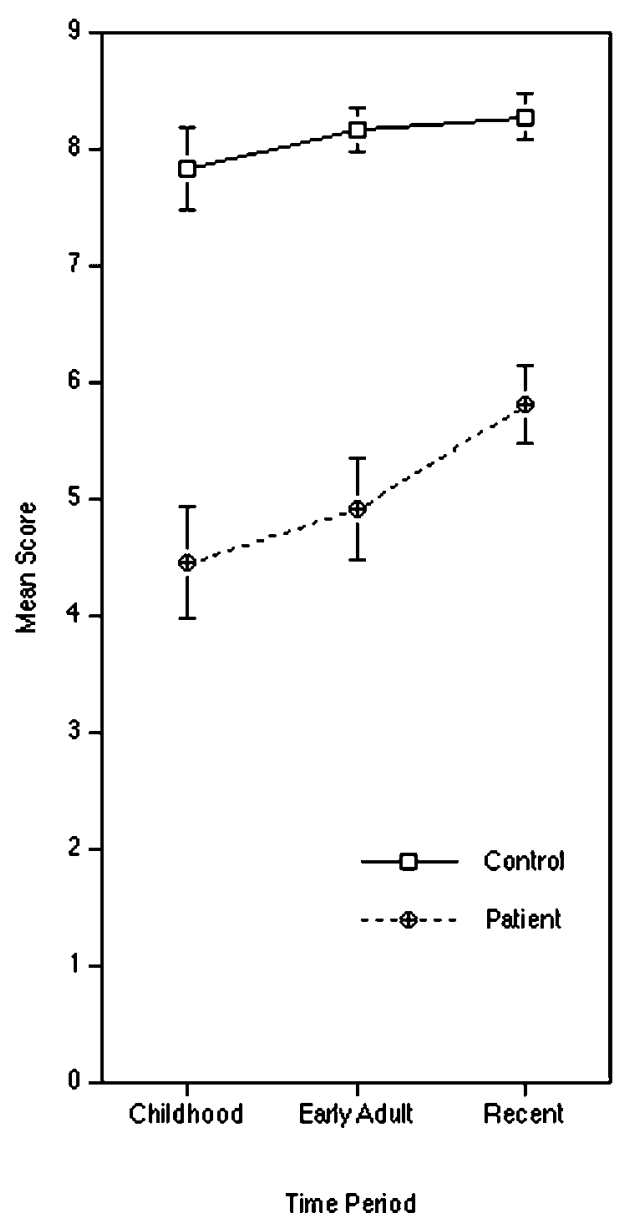

Figure 2. Autobiographical episodic memory performance. Mean scores on episodic components of AMI for control $(n=22)$ and patient $(n=25)$ groups. The maximum score is 9 per time period. Vertical lines depict SEM values.

arcsine transformation was performed before analysis to ensure homogeneity of variances. A 2 (group) $\times 3$ (time) $\times 2$ (type) ANOVA revealed a main effect of group $\left(F_{(1,45)}=55.81 ; p<\right.$ $0.001)$, with patients performing significantly more poorly than controls. There was also a main effect of type $\left(F_{(1,45)}=57\right.$. 74; $p<$ $0.001)$ and a significant type by group interaction $\left(F_{(1,45)}=47.38\right.$; $p<0.001)$. As shown in Figures 1 and 2, differences between patients and controls were confined to the episodic questions. There was a significant main effect of time (early, middle, and recent) $\left(F_{(2,90)}=6.90 ; p<0.002\right)$, with better performance for more recent periods. None of the interactions involving time was significant.

Separate analysis of the semantic items showed no significant effect of group $\left(F_{(1,45)}=0.885 ; p=0.352\right)$. There was a significant effect of time $\left(F_{(2,90)}=6.69 ; p=0.002\right)$, but no time $\times$ group interaction $\left(F_{(2,90)}=0.370 ; p=0.692\right)$. For the episodic section, significant effects were observed for group $\left(F_{(1,45)}=78.15 ; p<\right.$ $0.001)$ and time $\left(F_{(2,90)}=3.23 ; p<0.04\right)$, but the interaction was not significant $\left(F_{(2,90)}=0.971 ; p=0.382\right)$.

\section{Additional analyses}

Performance on the episodic component was evaluated further in the patient group to ascertain whether there were significant differences according to clinical variables, including age of onset of epilepsy, preoperative versus postoperative status, presence of medial temporal sclerosis by MRI, and affected hemisphere. None of these analyses revealed any significant main effects or interactions (with time period). Given the nature of the patient population, in which age of onset of epilepsy ranged from 0 to 36 years, it was also possible to examine recall of episodes occurring before and after

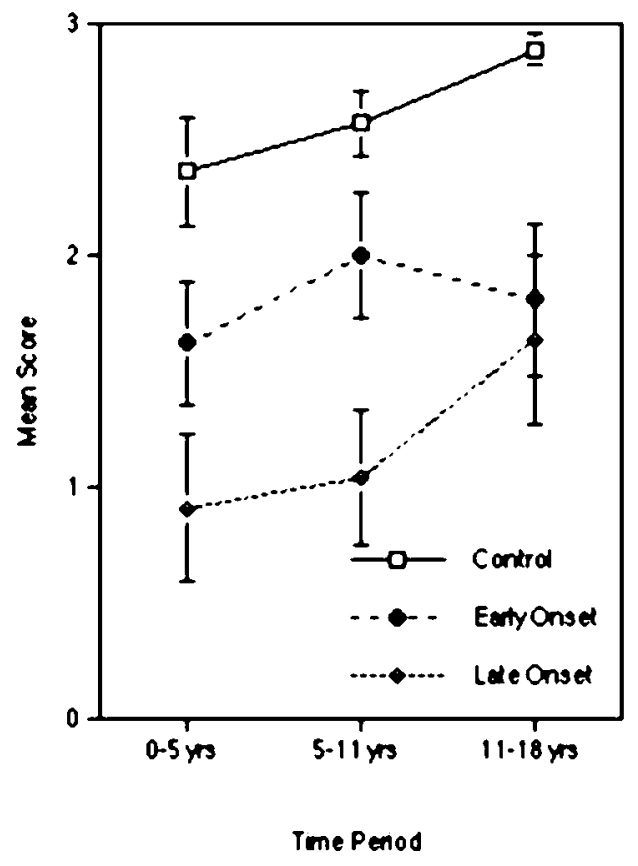

Figure 3. Autobiographical episodic memory performance during earliest time periods. Mean scores on episodic components of AMI for control $(n=$ 22 ), late seizure onset $(n=11)$, and early seizure onset $(n=8)$. Late seizure onset describes patients who reported first seizures after age 18, early seizure onset describes patients who reported first seizures before age 5 . The maximum score is 3 per time period.

the onset of recurrent seizures using the three earliest time periods $(0-5,5-11$, and $11-18$ years) from the AMI. Eight patients had seizures beginning before age 5 (mean age of onset, 3.5), and 11 patients had recurrent seizures beginning after age 18 (mean age of onset, 24.5 years). There was no significant differences $\left(F_{(1,17)}=\right.$ $2.78 ; p>0.11)$ in recall scores for autobiographical episodes across time periods for these two patient subgroups and no significant interactions with time (Fig. 3).

\section{DISCUSSION}

There are three key features of our finding of remote memory impairment in patients with temporal lobe epilepsy: defective retrieval of episodic memories with sparing of personal semantic memories, lack of a temporal gradient to the episodic memory deficit, and a duration that extended to early childhood. These results are consistent with the MTT of Nadel and Moscovitch (1997) but difficult to reconcile with consolidation theory (Scoville and Milner, 1957; Squire and Alvarez, 1995).

Our TLE patients exhibited no difficulties with recall of personal semantic memories despite profound impairment in recall of autobiographical episodes. Indeed, both patients and controls could recall semantic information readily even for the most recent time periods, indicating that such information is accessed easily. MTT suggests that personal semantic memories are more resistant to hippocampal damage because of multiple traces established through many retrieval attempts in the past and/or the evolution of independent semantic traces via the same mechanism. In contrast, consolidation theory proposes that hippocampal damage should affect episodic and semantic memories similarly, unless differential rehearsal can account for the relative sparing of semantic memories. Of course, this "add on" to consolidation theory regarding differential rehearsal is one of the processes by which MTT suggests semantic memories become resistant to disruption (for further discussion, see Moscovitch and Nadel, 1998, 1999; Nadel and Moscovitch, 1998).

There was no evidence of a temporal gradient in remote memory loss for personal episodes, with both patients and controls demonstrating better retrieval of more recent memories relative to more 
remote ones. The absence of a temporal gradient is not compatible with consolidation theory, which is founded on the principle of differential sparing of remote memories. However, this pattern can be accommodated by MTT if one assumes that episodic memories are not multiply reproduced in all their detail. Although the gist of the episode may be reproduced, the complete episode will leave few traces, and thus retrieval remains dependent on the hippocampal complex, which includes the hippocampal formation and perihippocampal structures (parahipocampal, perirhinal, and entorhinal cortex). Conceivably, our patients were attempting to retrieve unique experiences that had not been subject to many previous retrieval attempts from each time period, and thus no temporal gradient would be expected.

Review of the literature on retrograde amnesia indicates that the existence of a temporal gradient and its length depends on the extent of damage to the hippocampal complex; the greater the damage, the more severe and extensive the retrograde loss (Nadel and Moscovitch, 1997; Fujii et al., 2000). According to MTT, partial damage to this region results in temporally graded RA, because the multiple traces for more remote memories make them more resistant to loss. Complete damage results in loss of all episodic memories and an inability to retrieve more "vulnerable" semantic information that had been weakly encoded. This general pattern is also demonstrated in the RA of patients described by Squire and his colleagues (McKinnon and Squire, 1989; RempelClower et al., 1996; Reed and Squire, 1998). Lesions restricted to the hippocampus proper result in minimal RA, whereas more extensive lesions produce an RA for a decade or more before injury, with complete hippocampal complex lesions leading to complete loss of episodic memories. Of interest, our data do not reveal any association between the measures we used to assess the degree of medial temporal damage/dysfunction and the extent of remote memory impairment. The measures of hippocampal damage included age of onset (an indirect measure) and presence of hippocampal sclerosis (a direct measure).

Alternatively, it is conceivable that damage to other temporal lobe structures, such as the temporal pole (Markowitsch, 1995) is crucial in producing extensive RA. This is an unlikely explanation for our data, because there was no difference between postoperative patients, all of whom had the temporal pole resected, and those still awaiting surgery, whose temporal pole was presumed to be intact. We cannot discount the possibility, however, that seizure activity may have affected the temporal pole in all patients and reduced its function. Similarly, whereas damage or dysfunction in other temporal neocortical regions cannot be ruled out in our patients, the common region affected is the hippocampal complex.

To our knowledge, no studies have been published that report performance by TLE patients on the AMI, although similar findings of relative impairment on the episodic component have been described in a small sample of postoperative patients (O'Connor et al., 1999). Kopelman et al. (1989) reported that amnesic participants were impaired on both semantic and episodic portions of the test and that they showed a shallow temporal gradient, with partial sparing of more remote memories. Of interest, they found that age influenced the slope of the gradient, with young amnesics (age, <40 years) showing a relatively flat gradient and older amnesics (age, $>50$ ) demonstrating a more marked gradient. We note that the majority of our TLE patients were under age 50. In a recent study, Kopelman et al. (1999) reported a relatively flat temporal gradient for the recall of both personal semantic facts and autobiographical incidents by herpes encephalitis patients with extensive bilateral damage to the temporal lobes. However, as Kapur (1999) argued in his extensive review of retrograde amnesia, there are a number of studies documenting dissociations between episodic remote memories and related personal semantic memories. Because unilateral TLE patients in our study do not show impairment on the personal semantic questions, it may be argued that semantic memories are dually represented in both hemispheres and that bilateral damage is necessary to show such impairment. For episodic memories, however, different aspects may be represented in each hemisphere, making them more vulnerable to loss in the event of unilateral damage. This hypothesis may explain why patients can recall some episodic memories from a specific time period but not others.

It should be noted that the results reported in this study do not replicate those reported in a similar study by Barr et al. (1990). They examined patients who had undergone unilateral temporal resections for epilepsy on an extensive battery of remote memory tests, one of which is described as an "exhaustive array of questions concerning various life events and circumstances" that appears to tap similar episodic and semantic personal memories as the AMI. They found that only left TLE patients showed remote autobiographical memory impairments, whereas we found that both left and right TLE patients were impaired. A number of reasons can account for this discrepancy, among them, differences in the types of remote memory tests used as well as possible differences in the extent of lesions to the right and left temporal lobes.

One concern in assessing remote memory in patients with epilepsy is the difficulty in precisely dating the onset of the critical damage and thus providing an unequivocal temporal landmark for retrograde amnesia. Clearly, using the time of excision as this landmark is inadequate. Significant hippocampal damage is found in many patients with TLE before resective surgery (Miller et al., 1993), and the removal of a nonfunctional hippocampus may not produce any additional anterograde memory defect (Hermann et al., 1994). Our analysis of the episodic memory data according to age of onset of epilepsy speaks to this issue. We found the same extent and pattern of memory loss in the earliest time periods (ages $0-18$ ) for patients whose seizures began before age five and those whose seizures began after age 18. This finding suggests that the impairment in patients with late onset and likely those with early onset is retrograde in nature. Although the possibility of defective encoding in both groups cannot be discounted (i.e., even those with later onset may have had damaged medial temporal tissue before experiencing recurrent seizures), the absence of even a trend in the data for poorer performance by the early onset group weakens this conclusion.

In summary, findings from this study have helped to characterize remote memory in patients with temporal lobe epilepsy. Patients with unilateral temporal lobe pathology show greater loss of episodic than semantic autobiographical memories, as measured by the Kopelman AMI. Furthermore, this loss encompasses all time periods equally, extending even to early childhood. Findings from this study are generally consistent with the multiple trace theory of hippocampal function in memory and incompatible with the predictions of consolidation theory.

\section{REFERENCES}

Barr WB, Goldberg E, Wasserstein J, Novelly RA (1990) Retrograde amnesia following unilateral temporal lobectomy. Neuropsychologia 28:243-255.

Burnham WH (1903) Retroactive amnesia: illustrative cases and a tentative explanation. Am J Psychol 14:382-396.

Fujii T, Moscovitch M, Nadel L (2000) Memory consolidation, retrograde amnesia and the temporal lobes. In: Handbook of neuropsychology, Vol 2 (Cermak LS, ed), pp 199-226. Amsterdam: Elsevier.

Hermann BP, Wyler AR, Somes G, Duhan C, Berry AD, Clement L (1994) Declarative memory following anterior temporal lobectomy in humans. Behav Neurosci 108:3-10.

Howell DC (1997) Statistical methods for psychology. Belmont, CA: Wadsworth.

Kapur N (1999) Syndromes of retrograde amnesia: a conceptual and empirical synthesis. Psychol Bull 125:800-825.

Kopelman MD, Wilson BA, Baddeley AD (1989) The autobiographical memory interview: a new assessment of autobiographical and personal semantic memory in amnesic patients. J Clin Exp Neuropsychol 11:724-744.

Kopelman MD, Stanhope N, Kingsley D (1999) Retrograde amnesia in patients with diencephalic, temporal lobe or frontal lesions. Neuropsychologia 37:939-958.

Markowitsch HJ (1995) Which brain regions are critically involved in the retrieval of old episodic memories? Brain Res Rev 21:117-127.

McKinnon DF, Squire LR (1989) Autobiographical memory and amnesia. Psychobiology 17:247-256.

Miller LA, Munoz DG, Finmore M (1993) Hippocampal sclerosis and human memory. Arch Neurol 50:391-394.

Moscovitch M, Nadel L (1998) Consolidation and the hippocampal com- 
plex revisited: in defense of the multiple-trace model—discussion point. Curr Opin Neurobiol 8:297-300.

Moscovitch M, Nadel L (1999) Multiple-trace theory and semantic dementia: response to KS Graham. Trends Cognit Sci 3:87-89.

Nadel L, Moscovitch M (1997) Memory consolidation, retrograde amnesia and the hippocampal complex. Curr Opin Neurobiol 7:217-227.

Nadel L, Moscovitch M (1998) Hippocampal contributions to cortical plasticity. Neuropharmacology 37:431-439.

O'Connor M, Morin M, Verfaellie M, Greenblatt D, Doherty R, Cahn G, Schomer D (1999) Performance of temporal lobectomy patients on tests of remote memory. J Int Neuropsychol Soc 5:117.

Reed JM, Squire LR (1998) Retrograde amnesia for facts and events findings from four new cases. J Neurosci 18:3943-3954.
Rempel-Clower N, Zola SM, Squire LR, Amaral DG (1996) Three cases of enduring memory impairment after bilateral damage limited to the hippocampal formation. J Neurosci 16:5233-5255.

Ribot T (1882) Diseases of memory. New York: Appleton.

Sanders HI, Warrington EK (1971) Memory for remote events in amnesic patients. Brain 94:661-668.

Scoville WB, Milner B (1957) Loss of recent memory after bilateral hippocampal lesions. J Neurol Neurosurg Psychiat 20:11-21.

Squire LR (1992) Memory and the hippocampus: a synthesis from findings with rats, monkeys, and humans. Psychol Rev 99:195-231.

Squire LR, Alvarez P (1995) Retrograde amnesia and memory consolidation: a neurobiological perspective. Curr Opin Neurobiol 5:167-177. 